\title{
Traveling Electric Field Conveyor for Contactless Manipulation of Microparts
}

\author{
Felix M. Moesner ${ }^{1,2)}$ and Toshiro Higuchi ${ }^{2,3)}$ \\ 1) Swiss Federal Institute of Technology Zurich (ETHZ) - Institute of Robotics \\ 8092 Zurich, Switzerland•Email: moesner@ifr.mavt.ethz.ch \\ 2) Kanagawa Academy of Science and Technology • Higuchi 'Ultimate Mechatronics' Project \\ KSP Bldg., East 405, 3-2-1 Sakado, Takatsu-ku, Kawasaki 213, Japan• Fax: +81 448192095 \\ 3) University of Tokyo $\cdot$ Department of Precision Machinery Engineering \\ Hongo 7-3-1, Bunkyo, Tokyo 113, Japan•Email: higuchi@intellect.pe.u-tokyo.ac.jp
}

\begin{abstract}
In an earlier paper by the authors [1], downscaled devices for microparts handling utilizing an ac electric field boundary wave were proposed. Devices that instantly generate contactless microparts driving forces through electric field creation have been designed and fabricated. In a further attempt, the mechanisms behind microparts conveyance are here subsequently validated in experiments and simulations.

Particles as micropart substitute are actuated. On a thin protecting insulation-film above a series of encased and insulated parallel field electrodes, particles become either triboelectrically or induction charged through the application of balanced multi-phase voltages. The created non-uniform traveling field-wave conveys the charged particles perpendicular to the electrodes confined in potential wells from electrode to electrode. A series of particle materials with diameters up to $400 \mu \mathrm{m}$ has been examined; metal, glass, and plastic spheres showed the best performances. Simulations of the potential distribution underline the experimental findings on the electric panel and dots device. One further result, which could have been shown by experiments, is the preoscillation of a moving particle caused by gravity.
\end{abstract}

\section{INTRODUCTION}

Today's industrial world offers several techniques for micropart-handling, each being fitted for a specific objective in the process and also taking advantage of an explicit property of the microparts. These handling methods include classic pneumatic and hydraulic transportation by regulated carrier gas or liquid flow. Particles as microparts are conveyed in bulk in long pipes [2]. This purpose-oriented guidance of a mass of particles to a fixed target container renders micromanipulation of single objects nearly impossible. Similarly, transportation by surface vibrations can carry a bulk of particles to a fixed spot, too. These apparatuses form the group of parts feeder which are conceived purely for mass transportation and not for controlled micromanipulation of objects.
In another consideration, the existence or non-existence of a specific particle property may also be set as conveyance trigger. As an example, [3] demonstrates the use of magnetic fields to manipulate fine magnetic particles. High-gradient magnetic separation makes even the effective handling of very weakly magnetic micron-sized particles possible [4]. The advantage lies in the property-oriented separation of particles into magnetic and non-magnetic groups. However, this technique is inapplicable in the handling of purely nonmagnetic particles.

In addition to above methods, it is conceivable to use microgrippers for single particle manipulation. The goal of these systems is to handle and assemble micro structures. $[5,6]$ describe a nanorobot system for automated microparts manipulation with a resolution in the order of a nanometer. This goal-oriented method allows manipulation of single particles despite their different properties.

Some methods make use of focused laser beams [7]. The produced optical pressure which is sufficient to confine fine particles or cells in a gradient trap permits 3D micromanipulation. A different method employs ultrasonic standing wave fields for particle micromanipulation [8]. Particles are confined in acoustic radiation pressure traps. Both methods are contactless and work preferably in a liquid environment.

Above listed methods are related by the common purpose of particle handling. However, they are all together restricted to a particular range of handling abilities, such as bulk transportation, discrete handling or property-oriented manipulation. With the appropriate use of forces generated by an electric field however, it is possible to create very compact devices for particle handling, possessing advantages over the aforementioned methods and therefore providing an interesting alternative. 
The method of using electric fields for particle manipulation has already been outlined in various papers and proven in applications. In the early seventies, the electric curtain [9] was the first linear transportation system to drive fine particles in a state of continuous levitation with help of ac electric fields. Based on the same method of dynamic field waves, both $[10,11]$ developed particle transportation devices with structural wavelength on the order of a millimeter. Coarse, bi-directional transportation of fine particles is the result of these methods which is now being further improved by an extra attribute - precise and smooth particle handling in multiple directions on an open surface and in three practical environments: atmosphere, dielectric liquid and vacuum.

The focus of this paper lies on experiments and simulations of systems for particle handling in the interlayer of micro- and macro-world by making use of traveling electric fields as the contactless particle conveyor. The energizing electrodes integrated in microconveyors (expression for particle manipulation electric devices) are situated at an equal gap to each other and are of different designs, such as parallel and dotted conductors.

One goal is to overcome fluctuating disturbances during the particle manipulation process, yielding a smooth transit over the surface. The effective actuation characteristic of a particle is analyzed by both high speed camera and scanning electrode microscope (SEM).

\section{Traveling Wave and Particle Transportation}

Different microconveyors for particle microhandling using an ac electric field wave are proposed by $[1,12]$. Balanced, multi-phase high voltages from a programmable source are supplied to a series of encased, parallel electrodes which are covered and protected against electric sparkovers by a thin insulating film. Upon the activation of the electrodes, a traveling electric field is created around the electrodes which is transiently varying in sync with the applied voltage phase.

Particles on the thin insulating film above the parallel field electrodes become charged through the application of voltages to the electrodes. Dynamic forces of this traveling field-wave overcome adhesion and gravitational forces acting on the charged particles and actuate them in a direction in the plane perpendicular to the electrodes in potential wells from electrode to electrode.

The electric field strength and its resulting force acting on the particle depend on the applied voltage. Consequently, regulation of the voltage amplitude offers a means to tune the force on the particles. Further, parti- cles produce an overall conveyance velocity in direct proportion to the frequency of the electric field and the electrode gap.

A multi-channel, programmable, high voltage supply is connected to the microconveyor, where every sixth electrode belongs to the same phase state: $a, b, c, d, e$, or $f$. Particle transportation occurs with the variation of the sequence, i.e. the particle synchronously moves with one phase.

\section{Particle Charging}

Particles on the thin insulating film interface above the encased field electrodes become, either triboelectrically charged through contact friction for non-conductive objects when placed on the surface or induction charged through the application of voltages to the electrodes for conductive objects [13]. Fixation of durable charge onto particles is only feasible when the particulate objects are kept isolated from ground. Consequently, any conductive and non-conductive particle material may maintain its acquired charge, if a sufficient insulation is guaranteed [14]. The requirement of complete insulation from earth is provided by the thin insulator film covering the electrodes. Activation of the electrodes induces a charge around the electrodes on the film surface [15] which is herein confirmed experimentally. The charge on the surface is then affecting the nearest conductive particle.

In the following considerations of the charge quantity on the particle surface, particles in the micrometer range are idealized to be spherical and effects from any molecular interactions or chemical reactions are neglected. It is assumed that the charge is distributed homogeneously on the surface.

For a conductive particle, the acquisition of the induction charge resides on the surface of the particle and is nearly instantaneous. The total charge $q(\mathrm{C})$ is the integration of the surface charge yielding [16]:

$$
q=\frac{\varepsilon_{0} \cdot \pi^{3}}{6} \cdot d^{2} \cdot E
$$

where $E$ is the electric field strength $(\mathrm{V} / \mathrm{m}), d$ is the diameter $(\mathrm{m})$ of the spherical particle, and $\varepsilon_{0}$ is the permittivity of free space $\left(8.8510^{-12} \mathrm{~F} / \mathrm{m}\right)$.

For a non-conductive particle, the main forms of dynamical triboelectric contacts are sliding, rolling, vibrating on the surface and impact charging [17]. The total charge acquired is dependent on various parameter such as force of contact and velocity of frictional motion. The most likely explanation of this phenomenon is that the temperature of the contact point affects the 
charge transfer [18]. The maximal charge that a triboelectrified particle can hold on its surface amounts to [19]:

$$
q=\varepsilon_{0} \cdot \pi \cdot d^{2} \cdot E_{B}
$$

where $E_{B}$ is the breakdown field strength $(\mathrm{V} / \mathrm{m})$ of air that reaches approx. $3 \mathrm{MV} / \mathrm{m}$. Charging of fine particles materials can typical achieve charge-to-mass ratios in the order of $10^{-4} \mathrm{C} / \mathrm{kg}$ [17]. During the process of frictional charging, contact charging (tunneling effect of electrons) may be present which results in charge redistribution. When two surfaces of different chemical nature are brought together, a gain of potential energy can be achieved, stimulating contact charging. The actual charge redistribution is usually described as a quantum mechanical tunneling effect through the potential barrier. The potential barrier of a metal particle may be caused by a thin layer of oxide covering the surface [17].

Besides the mechanism of charging, a second important aspect has to be considered, namely, the mechanism by which the particle materials concerned retain their charge. Usually, the charge imparted on a particle vanishes within a certain time $\tau$. This charge relaxation time $\tau$ is defined as the time needed to discharge a particle to a charge level of $37 \%$ of the initial charge $q_{0}$ [19]. It is calculated from

$$
q(t)=q_{0} \cdot \mathrm{e}^{-t / \tau}
$$

as

$$
\tau=\varepsilon_{0} \cdot \varepsilon \cdot \rho
$$

where $\varepsilon$ and $\rho$ are the relative permittivity and the electric resistivity $(\Omega \mathrm{m})$ of the surrounding media, respectively.

\section{iV. Simulation of Electric Field Potential Distribution With Finite Difference Method}

In experiments, microconveyors are typically used in multi-phase actuation modes. It is now of interest to obtain the potential distribution of the electric field on the microconveyor. The focus of the following potential simulation is restricted to the static case where timeinvariant samples of sine and rectangular voltages are applied to the electrodes.

$$
\nabla^{2} v=0
$$

Laplace's equation (5) may be used for finding the potential distribution in the region between two conductors, given the charge distribution on the surfaces of the conductors or the potentials of the conductors or a combination of the two. Since the potentials on the electrodes are known, this problem may be solved from the Laplace's equation, neglecting any space charge effects. Attention is directed towards seeking the solution of Laplace's equation within a finite region bounded by a closed curve on which a boundary condition is specified at every point. The solution for the electric potential is first obtained over a mesh of points called nodes. By using numerical techniques, potentials are then obtained at any other defined point in the model. The technique chosen here is the Finite Difference Method [20].

The solution accuracy of (5) depends on the mesh fineness. In the Finite Difference computer program running on a Sun Ultra 1 Creator, the mesh is regularly spaced with unity. The program itself runs on a Mathematica interpreter [21].

Traditionally, the Finite Difference Method is used for solving field problems with two-dimensional geometry represented by the two-dimensional Laplace's equation

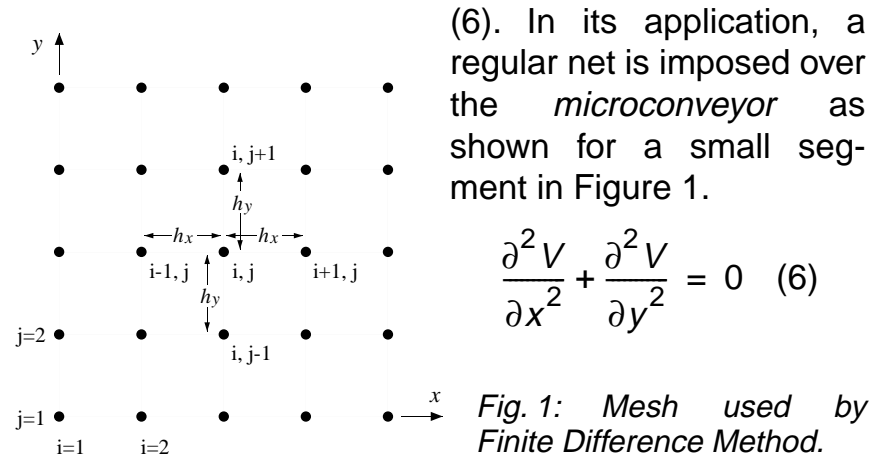

The nodal points at which a solution will be calculated are the intersections of the mesh lines. Now, the first stage is to derive a relationship between a node and its four closest neighbors. The potential at node (i, j) is supposed to be $V_{i, j}$. By Taylor's Theorem, if the spacing between node $(i, j)$ and the next node $(i+1, j)$ in the $x$ direction is $h_{x}, V_{\mathrm{i}+1, \mathrm{j}}$ may be expressed as the infinite series

$$
V_{i+1, j}=V_{i, j}+\frac{1}{1 !} \frac{\partial V}{\partial x} h_{x}+\frac{1}{2 !} \frac{\partial^{2} V}{\partial x^{2}} h_{x}^{2}+\ldots
$$

where the derivatives are those at node (i, j). Analogously, for node ( $\mathrm{i}-1, \mathrm{j})$ on the negative $x$ side of node ( $\mathrm{i}$, $j)$, the addition of these two equations results in

$$
v_{i+1, j}+v_{i-1, j}=2 v_{i, j}+\frac{\partial^{2} v}{\partial x^{2}} h_{x}^{2}+\delta
$$

where $\delta$ represents a small correction comprising the higher order terms which can be made as small as desired by diminishing $h_{x}$.

Thus 


$$
\frac{\partial^{2} V}{\partial x^{2}}=\frac{V_{i+1, j}+V_{i-1, j}-2 V_{i, j}}{h_{x}^{2}}
$$

is approximately true. Similarly, the equation is derived for a spacing of $h_{y}$ in the $y$ direction. Adding these two equations, it is found that (6) is formulated as: $\quad \nabla^{2} V=$

$$
\frac{V_{\mathrm{i}+1, \mathrm{j}}+V_{\mathrm{i}-1, \mathrm{j}}-2 V_{\mathrm{i}, \mathrm{j}}}{h_{x}^{2}}+\frac{V_{\mathrm{i}, \mathrm{j}+1}+V_{\mathrm{i}, \mathrm{j}-1}-2 V_{\mathrm{i}, \mathrm{j}}}{h_{y}^{2}}
$$

Setting $h_{x}=h_{y}=1$, i.e. the mesh spacing is unity in both coordinate directions, and regarding (6), the equation can be now written as

$$
V_{i, j}=\frac{1}{4}\left[V_{\mathrm{i}+1, \mathrm{j}}+V_{\mathrm{i}-1, \mathrm{j}}+V_{\mathrm{i}, \mathrm{j}+1}+V_{\mathrm{i}, \mathrm{j}-1}\right]
$$

In the model of the electric panel device, the boundary nodes and the nodes at the electrode positions already have potentials assigned to them. The potential of remaining internal nodes are initially set to zero and a table is made of all nodal potentials.

The Finite Difference mesh with equal spacings is now scanned sequentially, updating the tabulated value of the potential at each point according to (11). The scan takes place horizontally mesh line by mesh line and is repeated until errors $V_{\varepsilon}$ resulting from the finite-difference approximation are within specified limits.

$$
V_{\varepsilon}=\left|V_{i, j}-\frac{1}{4}\left[V_{i+1, j}+V_{i-1, j}+V_{i, j+1}+V_{i, j-1}\right]\right|
$$

This relaxation method produces fairly consistent values for the potential. For graphical presentation, a spline fit using nodal potential values is employed to generate smooth results.

Checker board pattern of Figure 2 shows particles being confined in spots. The potential distribution of Figure 3 gives a good agreement with a zoomed SEM image and also with the white frame of Figure 2. The illustration of Figure 4 reveals a good understanding, how the particles are trapped in the potential wells. This potential simulation is done within the handling surface.

In practice however, the cross-sectional configuration consists of layers with different permittivities. Two simplified example with two regions (air/epoxy) are illustrated in Figure 5 where the potential has been calculated by a supplementary equation formulated as followed:

$$
V_{i, j}=\frac{1}{4}\left[V_{i+1, j}+V_{i-1, j}+2 \frac{V_{i, j+1}+\frac{\varepsilon_{r 2}}{\varepsilon_{r 1}} V_{i, j-1}}{1+\frac{\varepsilon_{r 2}}{\varepsilon_{r 1}}}\right]
$$

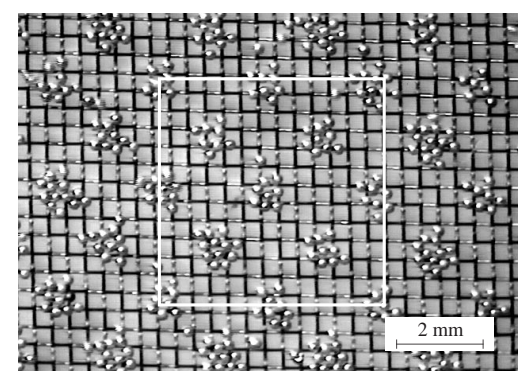

Fig. 2: Checker board pattern: Groups of particles are confined in potential wells.

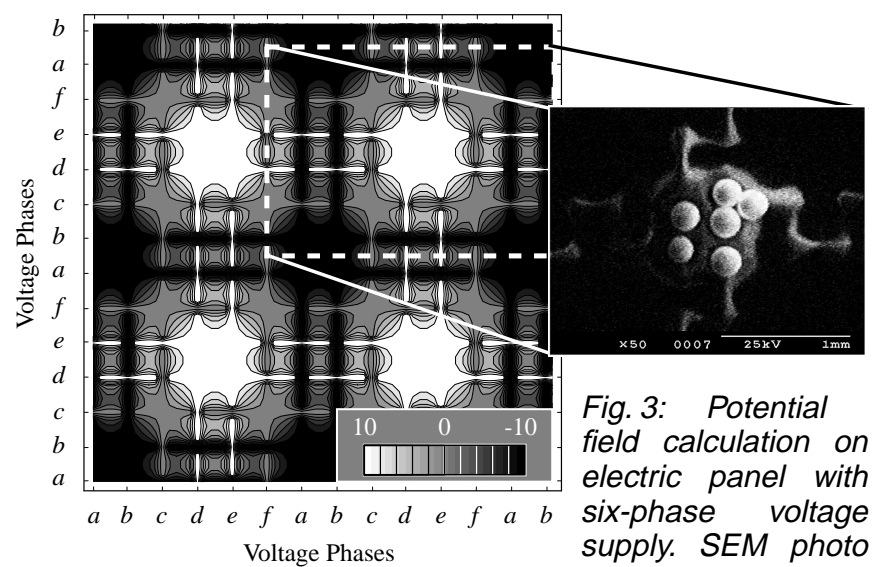

of an activated electric panel shows a potential well indicated by white color wherein a set of particles are confined.

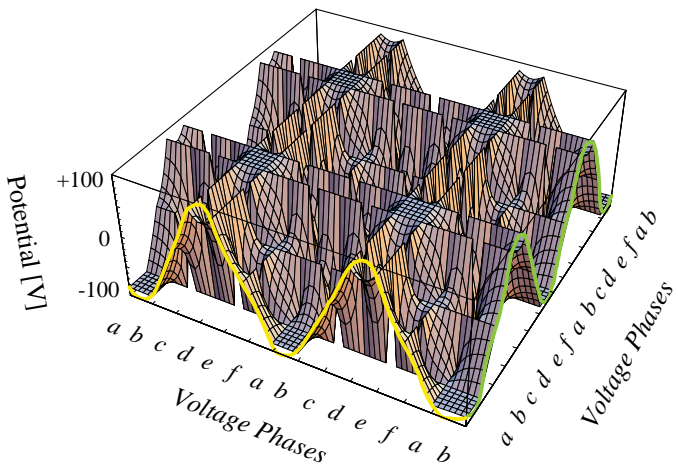

Fig. 4: Six-phase potential wells on an electric panel.
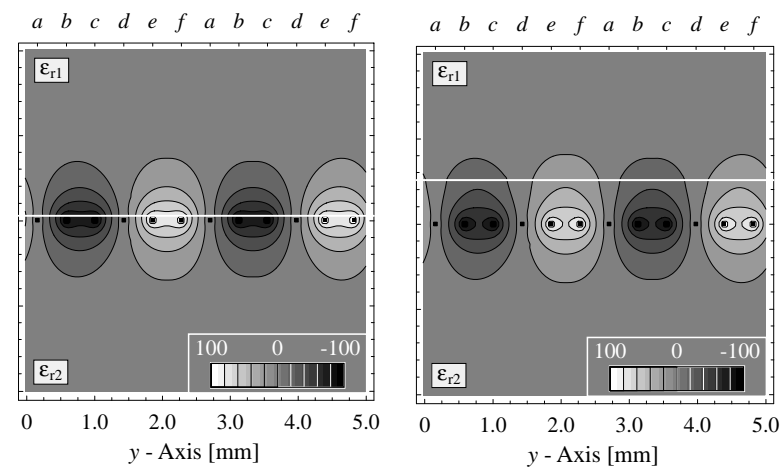

Fig. 5: Cross-sectional view of the inhomogenity produced by two spaces of different permittivity $\left(\varepsilon_{r 1}=1\right.$ for air and $\varepsilon_{r 2}=$ 3.7 for epoxy). The left and right media division appear $50 \mu \mathrm{m}$ and $600 \mu \mathrm{m}$ above the electrodes, respectively. 
Functioning as transfer element for the potential, the application of this equation is purely limited to the line of nodes right on the division of the two media. The potential of the nodes in the remaining region is obtained by (11).

\section{Microconveyor DeVices}

The microhandling of particles by the contactless traveling wave is mainly triggered by the following factors: the charge quantity on the particle surface, its charge relaxation time and the electric field strength. Microconveyors provide the basic means for a controllable electric field generation as well as for charge emission acquired by the particles.

The created dynamic electric field uses the electrodes as its primary guidance when it is formed around them. Consequently, the design of the electrodes is a key-element that influences the following factors:

- Particle size

- Transportability of particles

- Precision of particulate object positioning

- Total size of handling surface

To get the most benefit from the contactless conveyance capability, it is necessary to choose the appropriate electrode design. In the scope of this paper, two different microconveyors are used:

- The electric panel device permits multi-directional particle manipulation on a plane surface. Various techniques help to improve particle conveyance characteristics.

- The electric dots device allows fine and position-oriented manipulation of particles.

\section{Fabrication of Electric Panel Devices}

On an electric panel made with a single set of parallel electrodes, particles are bi-directionally (reversible directed) conveyed for the ideal case. By activation of a voltage sequence to the electrodes, particle are conveyed in direction with the traveling field. A reversal of the sequence inverts the direction of actuation. Introduction of a new set of perpendicular, parallel electrodes (forming a mesh) widens the manipulation possibilities to a multi-directional state. The mesh is woven with polyamide-imide (PAI) enameled electrodes.

The mesh electrodes are embedded in epoxy resin, covered by a thin insulating polyethylene terephthalate (PET) film (Mitsubishi Material Corp., Osaka) and supported by an acrylic substrate. PET as the cover film material functions as insulator and is further an element

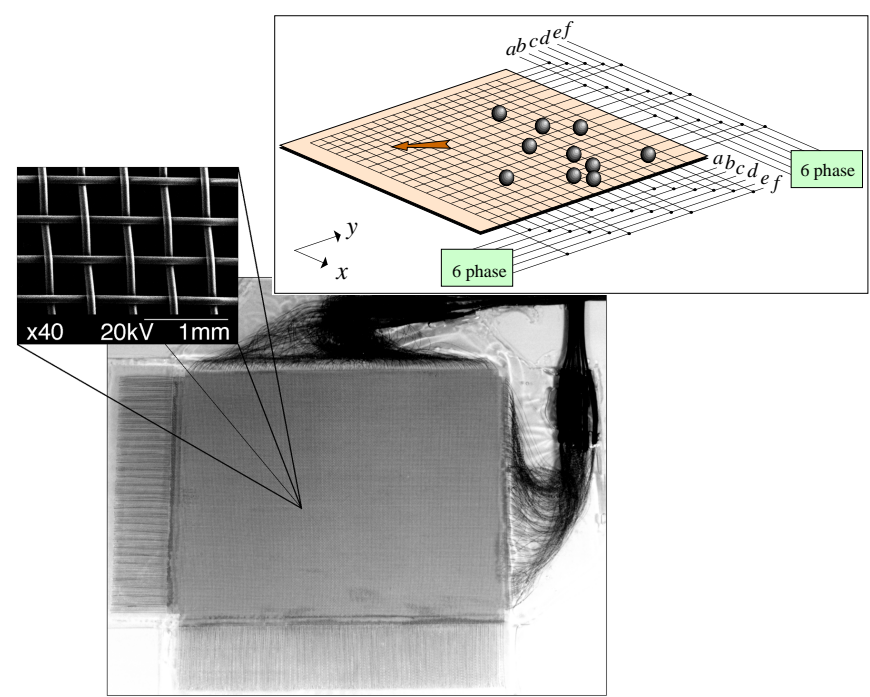

Fig. 6: Electric panel for multi-directional particle handling. A real electric panel is shown with magnified mesh electrodes. Highly insulated electrodes are woven to a mesh.

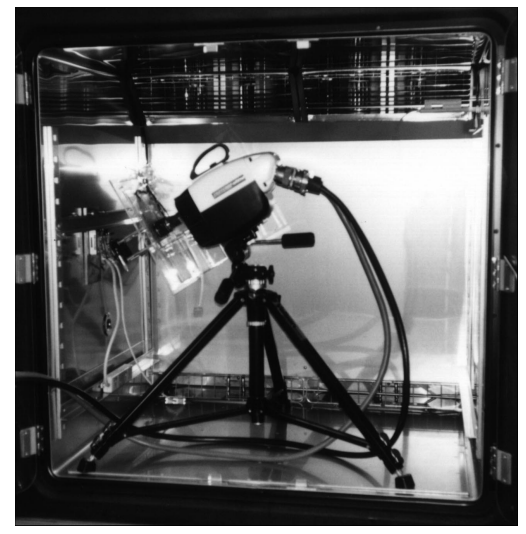

Fig. 7: Setup with high-speed camera in climate chamber allowing particle actuation to be recorded by high speed camera at inclinations up to $90^{\circ}$.

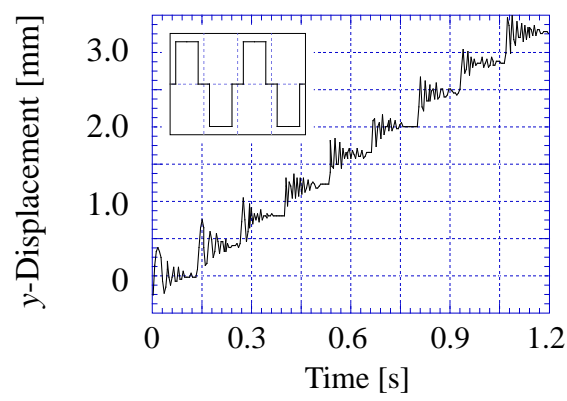

Fig. 8: Above: Recorded particle dynamics over the horizontal surface shown as step-trajectory.

Fig. 9: Graphs on the right: Step oscillation of a test particle. Pre-oscillation is caused by gravitational forces. The electric panel is increasingly tilted in each graph.
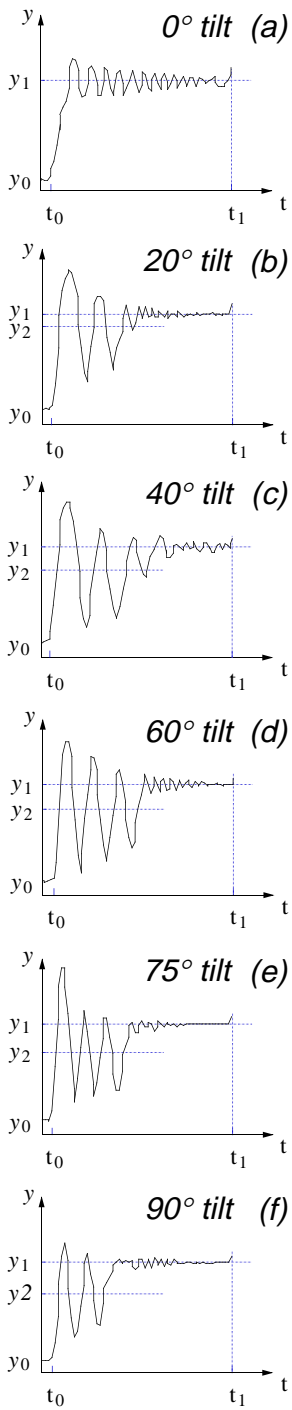
of the triboelectric material series [22], promoting frictional charging of particles. The illustration in Figure 6 shows the electric panel device with its successive electrode connection to their corresponding phases. The electrodes have an average center-to-center pitch length of $400 \mu \mathrm{m}$, a diameter of $50 \mu \mathrm{m}$ and are situated parallel to the surface at a depth range of $25-75 \mu \mathrm{m}$. For the fabrication process, epoxy resin $\left(\varepsilon_{r}=3.7\right.$; Plakiast, Nisshiri Corp., Japan) is used and the device is hardened in a repeating vacuum/pressure cycle until most air evacuated the sample.

\section{Particle Transportation with Influence of Gravitational Forces}

Experiments have demonstrated the ability of actuated particles to climb against gravity. An experimental setup for the investigation of these propulsive forces has been prepared. The electric panel device is affixed with nonconducting spacer rods onto a tilt-table that allows inclinations from horizontal position up to $90^{\circ}$. A high speed camera (Fastcam Ultima 13, Photron Corp., Japan) poses on a laboratory tripod and follows the preset gradient of the electric panel device as seen in Figure 7.

The ambient atmospheric condition is set to $20^{\circ} \mathrm{C}$ and $30 \% \mathrm{RH}$ inside the climate chamber (Tabai Espec Corp. Japan). Generally, before an experimentation series is started, the chamber needs preparation time of at least 3 hours in order to reach the preset atmospheric condition. Six-phase rectangular voltages are supplied to the electrodes at an amplitude of $700 \mathrm{~V}$ and at a frequency of $2 \mathrm{~Hz}$. The tracked conveyance technique [1] helps to facilitate the particle tracing process with the highspeed camera; reduced lateral fluctuations permit better focus on the influence of gravitational forces on actuated particles.

Test particles respond with a stepwise balanced oscillation when stepwise actuated on a horizontal electric panel device, as shown in Figures 8 and 9a. Now, measurements are taken at six angular inclinations of the electric panel device which are listed graphically in Figure 9. Each extract represents the oscillation of a 250 $\mu \mathrm{m}$ diameter iron particle, when proceeding one step to the next electrode in direction with the traveling field. The distance $\left|y_{1}-y_{0}\right|$ equals the $400 \mu \mathrm{m}$ electrode pitch and the oscillation of one step has a duration of 83.3 $m s$, which is equivalent to $\left|t_{1}-t_{0}\right|$ (Figure 9).

With an increasing tilt of the microconveyor, gravity begins to affect the particle dynamics, as seen in the graphs $9 \mathrm{~b}-\mathrm{f}$. The nature of the traveling field and the growing impact of gravity provokes a pre-oscillation with a lower center of oscillation, $y_{2}$. As the slope of the panel increases, this pre-oscillation center withdraws

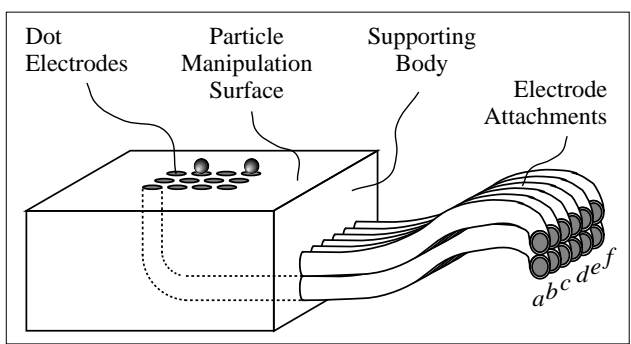

Fig. 10: Perspective of the electric dots device. The field electrodes are embedded in a epoxy resin body and connected to their phases.

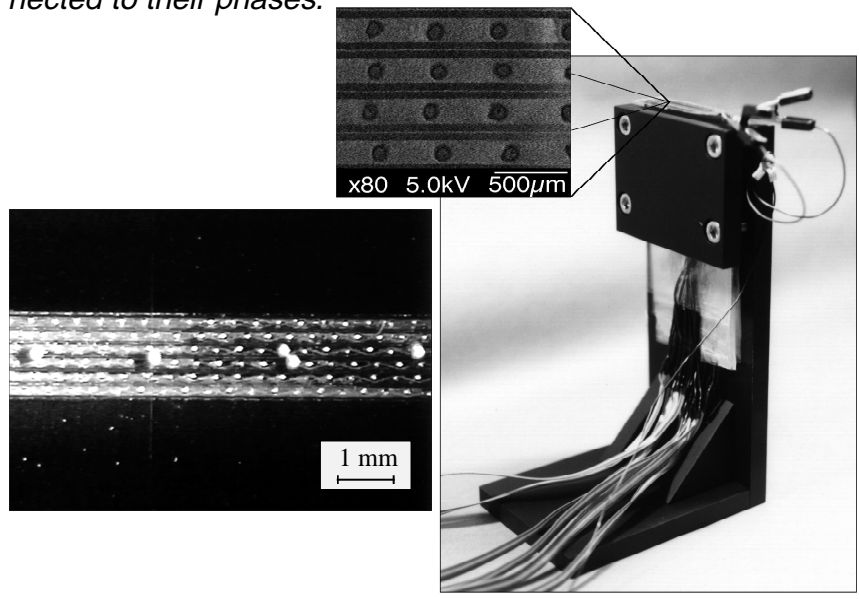

Fig. 11: Experimental setup of the electric dots device. The handling surface is at the top and magnified by a SEM image revealing its matrix dot electrodes. Left: Confined particle conveyance. Six-phase voltage wave transports particles along an array of electrodes.

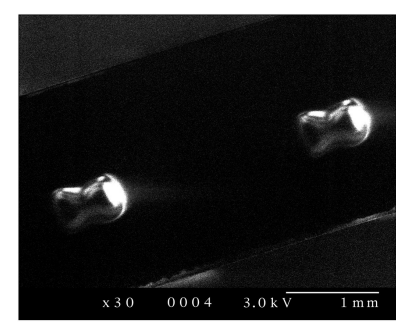

(a)
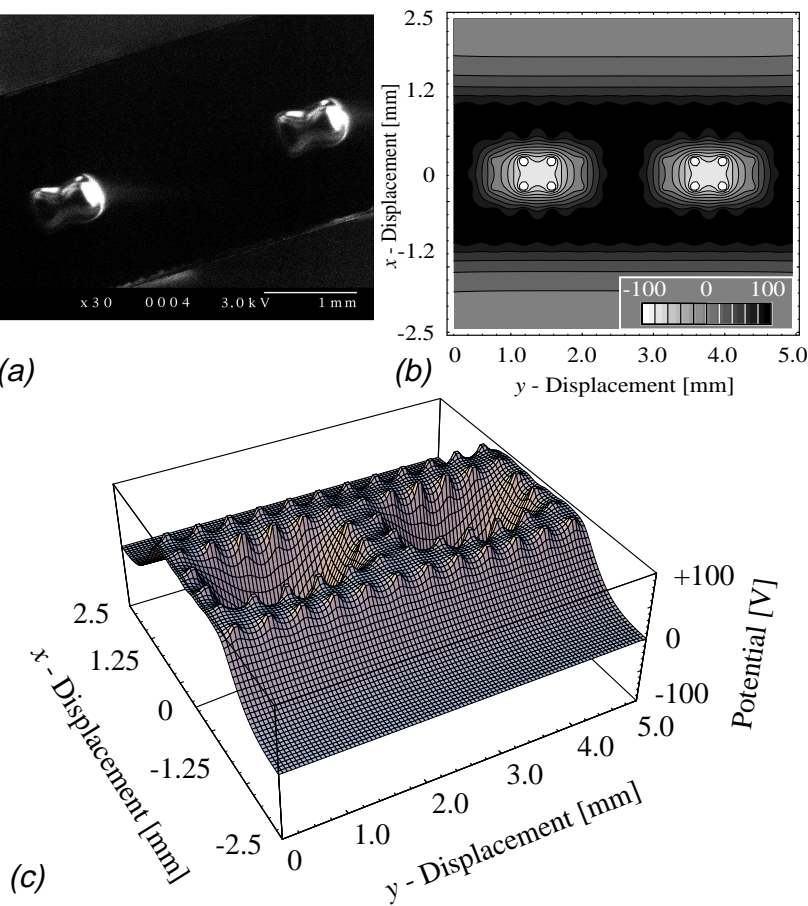

Fig. 12: SEM photo (a) and simulation (b,c) with analogous structural dimensions and six-phase voltage supply. The potential wells clearly appear in (c) as cavities. 
more and more from the next electrode and its keyoscillation. It is assumed that the wave-front of the traveling electric field attempts to move the particles along, but results in a retarding pre-oscillation. Particles remain in this pre-oscillation, until the electric field produces enough actuation force to lift them to the next electrode. At $90^{\circ}$, the resulting dynamics in $9 \mathrm{f}$ show that the center of oscillation is displaced by nearly a third of the total electrode pitch.

As a direct consequence, control of the voltage amplitude may produce an interesting tool to trigger selective particle handling under direct influence of gravity. Hereby, the mass of the particle is a major factor which may lead to mass-dependent sorting of particles.

\section{Fabrication of Electric Dots DeVICES}

The electric dots device consists of pinpoint electrodes which appear in a matrix formation at the manipulation surface as shown in Figure 10. Fabrication of the surface is done by grounding a plane normal to the wires and subsequently covering it with a thin film. The resultant dot electrodes have a center-to-center pitch length of $400 \mu \mathrm{m}$ and a diameter of $50 \mu \mathrm{m}$.

Generally, each electrode allows an independent voltage supply. With a large number of electrodes, however, the electronic part of the supply becomes more and more complex. Since the use of the rectangular supply is projected for maximal 16 channels, a reasonable electrode activation strategy is developed.

The design of the dots device incorporates 6 arrays of dot electrodes. The outer two rows are kept on dc provoking particle confinement and the inner two rows are on six-phase voltages producing particle actuation. This method resembles the tracked conveyance technique used to improve actuation quality on electric panel devices [1]. Figure 11 shows iron samples at a distance of six voltage phases moving in a direction along the center electrodes.

\section{Verification of SEM Image And Simulation}

An electric dots device with 6 arrays of electrodes is inserted into a modified SEM, externally supplied and observed in high vacuum condition. The charge distribution is analyzed and confirmed with computer simulations of same structural dimensions. Supplying sixphase rectangular voltages of $400 \mathrm{~V}$ amplitude to the two central electrode arrays and $+400 \mathrm{~V}$ dc to the peripheral electrodes, a charge distribution is measured on the device surface as depicted in the SEM micrograph of Figure 12a. The SEM image shows the manipulation surface from an oblique perspective.
Simulation of this situation is made by Finite Difference Method calculation. The resultant potential field distribution is shown in Figure 12b and its corresponding 3D graphical illustration in Figure 12c which permits a good illumination of potential wells by representing the potential magnitude in $z$-direction. Analogous to the SEM image, the positive and negative potentials are represented in $12 \mathrm{~b}$ as dark and light areas, respectively. A number of 9 equipotential levels are defined between minimum $-100 \mathrm{~V}$ and maximum $+100 \mathrm{~V}$ potential in the simulation.

All three results in Figure 12 show a good agreement of activation area shape and size. The two potential wells in 12c illustrate the mechanism behind confined particle transportation. The particle is trapped inside the well and actuated with the traveling wave. Peripheral electrodes create two potential walls and the traveling field generates moving separation walls forming the potential well.

\section{CONCLUSIONS}

In this paper, microconveyors have been fabricated for multi-directional microhandling of particulate objects actuated in a contactless fashion by non-uniform, timevarying electric fields. Two types of microconveyors, each meeting a different conveyance need have been experimentally validated.

The electric wave technique behind particle actuation is identical for both microconveyor types. Traveling electric waves are created around slim equidistant electrodes through application of time-varying, balanced, multiphase high voltages of $<1 \mathrm{kV}$ amplitude. The non-uniform electric field $(<57 \mathrm{kV} / \mathrm{cm} @<100 \mathrm{~Hz}$ ) is transiently altering and progressing in sync with the applied voltage phase. Particles, insulated by a thin film above the electrodes, become triboelectrically charged when non-conductive and induction charged when conductive, upon the activation of the electrodes. Dynamic forces of the electric field act against adhesion and gravitational forces of the charged particles and actuate them in a plane perpendicular to the electrodes in a stepwise fashion from electrode to electrode with the propagation speed of the wave.

Simulations of electric field potential distribution on activated devices are calculated with microconveyor dimensions and are successfully validated by charge distribution measurements in high vacuum $(\approx 0.01$ Torr $)$ with a modified scanning electron microscope.

The influence of gravity on particle conveyance dynamics is confirmed through experiments on an incrementally inclined manipulation surface. The existence of a 
pre-oscillation caused by the gravitational influence has been presented from experimental results.

Since these multi-environment microconveyors involve no moving machine parts, the use of the electric field can reduce overall energy consumption. Moreover, the handling surface is of virtually unlimited range. It is expected that the contactless ac electric field conveyor methods described in this paper will lead to new applications in various fields, such as parts feeders and manipulators for micro electro mechanical systems (MEMS). In the high frequency domain (>1 MHz), such contactless conveyors may contribute very promisingly to precise and efficient biological particulate handling needed in biomedical science and engineering.

\section{ACKNOWLEDGMENTS}

We wish to acknowledge the valuable collaboration of our colleagues S. Egawa, A. Fujita, J. Jin, T. Niino, S. Yashiro, and S.J. Woo. Highest appreciation is given to Bopp G. \& Co AG, Zurich Switzerland for their generous support, their contribution of excellent mesh material and their very efficient involvement in customized fabrication of mesh samples.

\section{REFERENCES}

[1] Moesner, F.M., and Higuchi, T., "Electrostatic Devices for Particle Micro-Handling," IEEE Industry Applications Society, 30th Annual Meeting, Orlando, vol. 2, 10 , 1995, pp. 1302-1309.

[2] Masuda, H., Komatsu, T., Mitsui, N., and linoya, K., "Electrification of Gas-Solid Suspensions Flowing In Steel and Insulating-Coated Pipes," Journal of Electrostatics, vol. 2, 1976 / 1977, pp. 341-350.

[3] Alward, U., and Imaino, W., "Magnetic Forces on Monocomponent Toner," IEEE Trans. Mag., vol. MAG-22, 1986, pp. 128-134.

[4] Oberteuffer, J.A., "High Gradient Magnetic Separation," IEEE Trans. Mag., vol. MAG-9, 1973, pp. 303-306.

[5] Codourey, A., Zesch, W., Büchi, R, and Siegwart, R., "A Robot System for Automated Handling in MicroWorld," IROS'95 IEEE/RSJ, Pittsburgh, vol. 3, August 1995, pp. 185-190.

[6] Zesch, W., Brunner, M., and Weber, A., "Vacuum Tool for Handling Microobjects with a Nanorobot," IEEE International Conference on Robotics and Automation, Albuquer-que, New Mexico, April 1997, pp. 1791-1766.
[7] Nishioka, M., Tanizoe, T., Katsura, S., and Mizuno, A., "Micro Manipulation of Cells and DNA Molecules," Journal of Electrostatics, vol. 35, 1995, pp. 83-91.

[8] Kozuka, T., Tuziuti, T., Mitome, H., and Fukuda, T. "Non-Contact Micromanipulation Using an Ultrasonic Standing Wave Field," Proceedings IEEE Micro Electro Mechanical Systems, San Diego, 1996, pp. 435-440.

[9] Masuda, S., Fujibayashi, K., and Ishida, K., "Elektrodynamisches Verhalten aufgeladener Aerosolteilchen im inhomogenen Wechselfeld und seine Anwendungsmöglichkeiten in der Staubtechnik," Staub-Reinhalt Luft, vol. 30, 1970, pp. 449-456.

[10] Melcher, J.R., Warren, E.P., and Kotwal, R.H., "Theory for Finite-Phase Traveling-Wave Boundary-Guided Transport of Triboelectrified Particles," IEEE Trans. on Industry Applications, vol. 25., no. 5, 1989, pp. 949-955.

[11] Schmidlin, F.W., "A New Nonlevitated Mode of Traveling Wave Toner Transport," IEEE Transactions on Industry Applications, vol. 27, no. 3, 1991, pp. 480-487.

[12] Moesner, F.M., and Higuchi, T., "Devices for Particle Handling by an ac Electric Field," Proceedings IEEE Micro Electro Mechanical Systems, 1995, pp. 66-71.

[13] Masuda, S., and Matsumoto, Y., "Contact-type Electric Curtain for Electrodynamical Control of Charged Dust Particles," Proc. 2nd Int.Conf.on Static Electricity, Frankfurt, 1973, Dechema-Monogr. No. 72, Nr. 1370-1409, Verlag Chemie, 1974, pp. 293-301.

[14] Koyano, K., Private Communication, Research Center for Advanced Science and Technology, University of Tokyo, email: koyano@lssl.rcast.u-tokyo.ac.jp, 1996.

[15] Niino, T., Egawa, S., Nishiguchi, N., and Higuchi, T., "Development of an Electrostatic Actuator Exceeding 10N Propulsive Force," Proceedings IEEE Micro Electro Mechanical Systems Workshop, Travemünde, Germany, 1992, pp. 122-127.

[16] Novick, V.J., Hummer, C.R., and Dunn, P.F., "Minimum dc Electric Field Requirements for removing Powder Layers drom a Conductive Surface," Journal of Applied Physics, vol. 65 (8), 1989, pp. 3242-3247.

[17] Chang, J., Kelly, A.J., and Crowley, J.M., "Handbook of Electrostatic Processes," Marcel Dekker, Inc., 1995.

[18] Cross, J., "Electrostatics: Principles, Problems and Applications," Adam Hilger, Bristol, 1987.

[19] Secker, P.E., "Instrumentation for Electrostatic Measurements," Journal of Electrostatics, vol. 16, 1984, pp. 1-19.

[20] Rao, N.N., "Elements of Engineering Electromagnetics," Prentice-Hall Inc., Fourth Edition, 1994.

[21] Wolfram, S., "Mathematica - A System for Doing Mathematics by Computer," Addison-Wesley Publ. Company, Second Edtion, 1991.

[22] Moore, A.D., "Electrostatics and its Applications," John Wiley \& Sons, 1973. 